\title{
Design of polymer-based antimicrobial hydrogels through physico-chemical transition
}

\author{
Emanuele Mauri $^{\mathrm{a}, 1}$, Davide Naso ${ }^{\mathrm{a}}$, Arianna Rossetti ${ }^{\mathrm{a}}$, Elisa Borghi ${ }^{\mathrm{b}}$, Emerenziana Ottaviano ${ }^{\mathrm{b}}$, \\ Gianmarco Griffini ${ }^{\mathrm{a}}$, Maurizio Masi ${ }^{\mathrm{a}}$, Alessandro Sacchetti ${ }^{\mathrm{a}, *}$, Filippo Rossi ${ }^{\mathrm{a}, *}$ \\ a Department of Chemistry, Materials and Chemical Engineering “Giulio Natta”, Politecnico di Milano, piazza Leonardo da Vinci 32, 20133 Milan, Italy \\ ${ }^{\mathrm{b}}$ Department of Health Sciences, Università degli Studi di Milano, via Di Rudinì 8, 20142 Milan, Italy
}

A R T I C L E I N F O

\section{Keywords:}

Antimicrobial

Drug delivery

Hydrogels

Polymer

Rheology

\begin{abstract}
A B S T R A C T
The antimicrobial activity represents a cornerstone in the development of biomaterials: it is a leading request in many areas, including biology, medicine, environment and industry. Over the years, different polymeric scaffolds are proposed as solutions, based on the encapsulation of metal ions/particles, antibacterial agents or antibiotics. However, the compliance with the biocompatibility criteria and the concentration of the active principles to avoid under- and over-dosing are being debated. In this work, we propose the synthesis of a versatile hydrogel using branched polyacrylic acid (carbomer 974P) and aliphatic polyetherdiamine (elastamine ${ }^{\circledR}$ ) through physico-chemical transition, able to show its ability to counteract the bacterial growth and infections thanks to the polymers used, that are not subjected to further chemical modifications. In particular, the antimicrobial activity is clearly demonstrated against Staphyloccoccus aureus and Candida albicans, two well-known opportunistic pathogens. Moreover, we discuss the hydrogel use as drug carrier to design a unique device able to combine the antibacterial/antimicrobial properties to the controlled drug delivery, as a promising tool for a wide range of biomedical applications.
\end{abstract}

\section{Introduction}

In the last years researchers have strongly focused their efforts in the development of antibacterial and antimicrobial materials. Although the study of antimicrobial field dates back about one hundred years, the design of polymeric systems able to exhibit efficient inhibition of bacterial infections is a discussed and thriving technology, that has become pivotal not only in biological fields, hospital and healthcare environments, but also in laboratory, marine and some industrial applications [1-4]. The direct use of antibiotic agents could be the approach to counteract many infections, but the main constraints are related to the potential environmental toxicity, the bacterial resistance, the shortterm antimicrobial activity and the proteolytic instability and degradation [5,6]. In addition, conventional antibiotics show problems about the solubility and overdose concentration range [7-9]. Therefore, the design of a biocompatible and efficient therapeutic delivery system, which can satisfy both the cytotoxicity issues and the antimicrobial criteria, is in high demand. Moreover, in biomedical applications, the loading of drugs or other active molecules over this kind of substrate represents a challenge to maximize the performance of the therapeutic cargo without inflammations and secondary side-effects during the medical treatment $[6,10,11]$. Scientific literature suggests different advanced antibacterial materials combining metal ions, natural compounds and modified polymers [12-14]. Among them, hydrogels are extensively studied as an alternative and promising tool due to their key properties. Defined as three-dimensional (3D) scaffolds composed by the chemical or physical entanglement of natural and/or synthetic polymer chains, hydrogels appear as suitable materials counteracting the microbial effects thanks to their biostability, biocompatibility and biomimetic physico-chemical properties, including swelling behavior $[15,16]$. Different strategies have emerged to develop hydrogels for antimicrobial applications: through the adsorption of antimicrobial agents $[17,18]$, the encapsulation of metal particles or polycationic groups [19-22], the material modification with covalent linkers to graft antibacterial peptides or synthetic compounds [23-25].

The use of metal nanoparticles, involving silver, gold, copper, zinc oxide, has to overcome two main constraints: the first one related to a high spatial dispersion to avoid the agglomeration within the gel scaffold, the second one about the compatibility and satisfying combination of an inorganic component with an organic environment [12,26].

\footnotetext{
* Corresponding authors.

E-mail addresses: alessandro.sacchetti@polimi.it (A. Sacchetti), filippo.rossi@polimi.it (F. Rossi).

${ }^{1}$ Present address: Department of Engineering, Università Campus Bio-Medico di Roma, via Alvaro del Portillo 21, 00128 Rome, Italy.
} 
Alternatively the chemical modification of antimicrobial agents through covalent bonds, exploiting the peptides amino and carboxyl terminal groups $[27,28]$, offers many advantages to achieve long-term antimicrobial activity, but with a special attention towards their structural integrity $[29,30]$. All these considerations could be skipped through the proposal of a hydrogel able to show antimicrobial features thanks to the starting polymers used in the sol/gel synthesis, without any further additions of antibacterial peptides, antibiotics or metal derivatives. In this work, we propose the synthesis of antimicrobial hydrogels via physico-chemical transition using two polymers: branched polyacrylic acid (carbomer 974P) and aliphatic polyetherdiamine $\left(\right.$ elastamine ${ }^{\circledast}$ ). The simple synthesis approach and the lack of compliance problems related to the biocompatibility and antimicrobial molecule dosage dependency in the final applications qualify this hydrogel able to conquer the vast issue of the traditional therapies and its promising application also in industrial fields. Finally, we have tested this hydrogel as a controlled drug delivery system. In the first casestudy, we have considered the release kinetics of two drug-mimetics: fluorescein sodium salt (SF) and rhodamine B (RhB) (commonly used as mimetic drugs [31]), entrapped by steric hindrance within the network meshes. In the second case-study, we have investigated the effect of a cleavable linker between the mimetic drug and the polymeric network using polyethylene glycol (PEG) modified ester to graft RhB: the cleavability of the ester linker allows a more prolonged and sustained drug release over time. These results show the promising use of the synthetized hydrogels not only in the antimicrobial field, but also as a therapeutic tool for the delivery and controlled release of active agents in biomedical applications, providing a potential solution to the design of a tool able to combine antibacterial/antimicrobial and therapeutic care.

\section{Materials and methods}

\subsection{Materials}

Hydrogel synthesis was performed using: carbomer 974P (MW $\cong 1 \mathrm{MDa}$, Fagron, The Netherlands) and elastamine ${ }^{\circledast}$ RE-2000 amine (MW $=2 \mathrm{kDa}$, Huntsman Corporation, Italy) as polyetherdiamine. Polyethylene glycol $(\mathrm{MW}=8 \mathrm{kDa})$ and all other chemicals involved in this work were purchased from Merck (Merck KGaA, Darmstadt, Germany). The materials were used as received, without further purification. Solvents were of analytical laboratory grade. Synthetized products containing fluorescein sodium salt or rhodamine B were stored at $4^{\circ} \mathrm{C}$ in dark, until their use.

\subsection{Synthesis of physical hydrogels}

The first step of the hydrogel design concerns the synthesis of the physical polymeric network. Carbomer 974P (140 mg, $1.94 \mathrm{mmol}$ of $\mathrm{COOH})$ and elastamine $\left(1200 \mathrm{mg}, 1.2 \mathrm{mmol}\right.$ of $\left.\mathrm{NH}_{2}\right)$ were independently dissolved in distilled water (respectively, $1.5 \mathrm{~mL}$ for carbomer and $8.5 \mathrm{~mL}$ for the polyetherdiamine). The resulting solutions were separately loaded into two syringes and the latter connected through a syringe mixing tube. Then, the polymeric solutions were mixed together for $2 \mathrm{~min}$ to obtain a homogeneous system. The physical mixture, representing the physical gel system, was placed in steel cylinders $(0.4 \mathrm{~mL}$ each and with the cylinder diameter of $1.1 \mathrm{~cm})$ and frozen at $-20^{\circ} \mathrm{C}$. Lastly, the molds were taken off and the final physical hydrogel configuration was obtained coming back to room temperature (r.t.). Referring to their nature, these hydrogels will be referred as physHG.

\subsection{Synthesis of chemical hydrogels via physico-chemical transition}

Physical hydrogels were freeze-dried and then subjected to microwave irradiation $(500 \mathrm{~W})$ for $25 \mathrm{~min}$ to induce the direct amidation of carbomer carboxyl groups with elastamine terminal $-\mathrm{NH}_{2}$, that gave rise to the formation of covalent linkers in the 3D hydrogel network. In this way, the microwave-assisted reaction allowed the transition from physical to chemical polymeric matrix. The resulting physico-chemical hydrogels (hereafter labeled chem-HG) were cooled down to r.t. and then stored at $4{ }^{\circ} \mathrm{C}$.

\subsection{Characterization techniques}

\subsubsection{FT-IR analysis}

FT-IR spectra were recorded using a Thermo Nexus 6700 spectrometer coupled to a Thermo Nicolet Continuum microscope equipped with a $15 \times$ Reflachromat Cassegrain objective, at room temperature in air in the $4000-500 \mathrm{~cm}^{-1}$ wavenumber range with 64 accumulated scans and at a resolution of $4 \mathrm{~cm}^{-1}$, using the $\mathrm{KBr}$ pellet technique for all samples.

\subsubsection{Rheology}

Hydrogels rheological properties were evaluated using a Rheometrics DSR200 rheometer with a $25 \mathrm{~mm}$ plate-cone configuration at $30^{\circ} \mathrm{C}$. Dynamic stress sweep (DSS) tests were performed in the range 5-300 Pa, at $1 \mathrm{~Hz}$. The oscillatory responses ( $\mathrm{G}^{\prime}$, elastic modulus and $\mathrm{G}^{\prime \prime}$, loss/viscous modulus) were determined at low values of strain over the frequency range $0.1-100 \mathrm{~Hz}$. The pseudoplastic behavior was also investigated. The behaviors of shear stress and viscosity as a function of shear rate were examined and the linearity of the viscoelastic properties was verified.

\subsubsection{Swelling behavior}

Physico-chemical hydrogel swelling was studied in distilled water, buffer phosphate saline (PBS) solution, at $\mathrm{pH}=10$ and at $\mathrm{pH}=3$. The first two conditions resemble a physiologic-like environment with a focal point related to the potential influence of salts in the responsive swelling behavior; the basic and acid environment mimic the conditions of inflammatory disorders. After the microwave-assisted synthesis, the hydrogel samples were weighed $\left(\mathrm{W}_{\mathrm{d}}\right)$ and poured into an excess of the corresponding neutral, alkaline or acid aqueous solution to achieve the complete swelling at $37^{\circ} \mathrm{C}$ under a $5 \% \mathrm{CO}_{2}$ atmosphere. Gravimetrical measures were recorded to estimate the swelling kinetics: the samples were removed from the aqueous solution at fixed time points (range $1 \mathrm{~h}-300 \mathrm{~h}$ ) and the surfaces were wiped with moistened filter paper in order to remove the excess solution, then they were weighed $\left(\mathrm{W}_{t}\right)$. The swelling ratio $\left(Q_{m}\right)$ was calculated according to the following equation:

$\mathrm{Q}_{\mathrm{m}}=\frac{\mathrm{W}_{\mathrm{t}}-\mathrm{W}_{\mathrm{d}}}{\mathrm{W}_{\mathrm{d}}} \cdot 100$

$\mathrm{W}_{\mathrm{t}}$ represents the weight of the wet hydrogel as a function of time, and $\mathrm{W}_{\mathrm{d}}$ is the weight of the dry sample.

\subsubsection{Scanning electron microscope (SEM) analysis}

Environmental SEM analysis was performed on gold sputtered samples at $10 \mathrm{kV}$ with Evo $50 \mathrm{EP}$ Instrumentation (Zeiss, Jena, Germany). To preserve the morphology of the physico-chemical hydrogel under complete swelling, freeze-drying (for $24 \mathrm{~h}$ ) was applied to remove all the liquid phase by sublimation. Due to the low operating values of temperature and pressure, the polymer chains were expected to retain the same conformation they had in wet conditions. Evaluations of the superficial and internal morphology of the investigated chem-HG samples (triplicate) were carried out.

\subsubsection{UV/vis spectroscopy}

The drug release studies were conducted through the evaluation of $\mathrm{SF}$ and RhB absorbance. It was measured by $\mathrm{Tecan}^{\circledR}$ Microplate Reader with UV/vis spectroscopy applying the Lambert-Beer method. 


\subsection{Antimicrobial analysis}

Hydrogels were placed in a 24-well-plate and sterilized under UV light for $30 \mathrm{~min}$. The reference strain Staphylococcus aureus ATCC 6538 and Candida albicans SC5314 were used for in vitro assays. Before each test, microbial strains were thaw from glycerol stock stored at $-80^{\circ} \mathrm{C}$ by seeding them on fresh Trypticase soy agar (TSA, for $S$. aureus) or Saboraud Dextrose agar (SAB, for C. albicans) and culturing them for $24 \mathrm{~h}$ at $37^{\circ} \mathrm{C}$. A single colony was harvested in phosphate buffer saline (PBS) to obtain a $0.5 \mathrm{McF}$ arland suspension (corresponding to about $10^{8} \mathrm{CFU} / \mathrm{mL}$ for $S$. aureus and $10^{6} \mathrm{CFU} / \mathrm{mL}$ for C. albicans). Serial dilutions were then performed in Trypticase soy broth (TSB, for $S$. aureus) or RPMI 1640 medium (for C. albicans) until the desired concentration $\left(10^{4}-10^{5} \mathrm{CFU} / \mathrm{mL}\right.$, respectively). Two $\mathrm{ml}$ of the suspension were added drop-by-drop to hydrogels, allowing gradual absorption. Positive growth controls, consisting of S. aureus ATCC 6538 and C. albicans SC5314 cultured without hydrogels, were run in parallel. After $24 \mathrm{~h}$ incubation at $37^{\circ} \mathrm{C}$, both supernatant and hydrogels were collected in 50 -mL tubes containing $18 \mathrm{~mL}$ of PBS and vigorously mixed to harvest microbial cells for CFU counting. Plates were incubated at $37^{\circ} \mathrm{C}$ and colonies were counted after $24 \mathrm{~h}$ of growth.

\subsection{Drug loading within hydrogels}

The drug mimetic loading was performed by adding SF or RhB in the phys-HG synthesis step. SF is a sodium salt like several drugs already listed and in water it is dissociated into fluorescein anion and sodium cation [32], whereas $\mathrm{RhB}$ is a neutral molecule at $\mathrm{pH}=7.4$ and slightly positive at acidic $\mathrm{pH}[33,34]$. In details, regarding SF studies, the traceable molecule $(10 \mathrm{mg})$ was dissolved into the elastamine solution $(1.5 \mathrm{~mL})$ to obtain a SF concentration of $6.67 \mathrm{mg} / \mathrm{mL}$; consequently, the resulting mixture was mixed with carbomer solution $(8.5 \mathrm{~mL})$ using the syringe mixing tube technique. Then, the gelling system was poured into steel cylinders $(0.4 \mathrm{~mL})$, frozen and lyophilized as discussed in the previous sections, and finally treated with microwave irradiation to obtain the final chem-HG containing SF. The final concentration of the drug mimetic was recorded $1 \mathrm{mg} / \mathrm{mL}$. The same approach was developed to synthetize hydrogels encapsulating RhB: in this case, the RhB concentration in elastamine solution was $1 \mathrm{mg} / \mathrm{mL}$ due to the water solubility constraints [35-37], and the final concentration in the chemHG was calculated equal to $0.15 \mathrm{mg} / \mathrm{mL}$.

\subsection{Synthesis of hydrogels with drug modified ester}

The study of the antimicrobial hydrogels as tunable drug delivery systems characterized by a cleavable RhB linkage was planned using a chemoselective functionalized PEG with ester bond grafting RhB, synthetized in our previous work [38]. Following a procedure similar to the one discussed in the SF and RhB loading by steric hindrance, PEG modified ester-RhB $(32 \mathrm{mg}$ ) and elastamine $(960 \mathrm{mg})$ were dissolved in distilled water $(1.2 \mathrm{~mL})$ and then mixed with the carbomer aqueous solution (112 mg in $6.8 \mathrm{~mL}$ of distilled water) using the two syringes and the mixing connection-tube.

Then, the resulting homogeneous mixture was partitioned in $0.4 \mathrm{~mL}$ in each steel cylinder, freeze-dryed and subjected to the electromagnetic stimulation by microwave $(25 \mathrm{~min})$ to give rise to the final amide-based hydrogel scaffold. These hydrogels are named chem-HGester-RhB, with regard to the presence of the cleavable linker between the mimetic drug and the polymeric network.

\subsection{In vitro drug release}

Chem-HG entrapping SF and RhB and chem-HG-ester-RhB were individually submerged in $2 \mathrm{~mL}$ of water-based solution (physiologic $\mathrm{pH}$ ) and stored at $37^{\circ} \mathrm{C}$, under a $5 \% \mathrm{CO}_{2}$ atmosphere. At defined time points, for each sample, a volume of $100 \mu \mathrm{L}$ was collected and poured into a 96-wells plate; each withdrawal was made in triplicate, removing overall $300 \mu \mathrm{L}$. The aliquots were used in UV/vis measurements and they were substituted in the release media with $300 \mu \mathrm{L}$ of distilled water in order to keep the volume invariable and preserve the diffusion regime. The $\mathrm{SF}$ and $\mathrm{RhB}$ released amounts were respectively measured via spectrophotometer at $\lambda=485 \mathrm{~nm}$ and $\lambda=570 \mathrm{~nm}$ and quantified referring to the standard calibration curve of the two drug mimetics. The percentage of the released molecule was defined as the ratio of the released absolute amount in the aqueous media to the sum of the total amounts of the released and unreleased SF or RhB. Three samples per type were used in this experimental procedure and the results were averaged.

\subsection{Statistical analysis}

The experimental data related to swelling behavior and drug release were analyzed using Analysis of Variance (ANOVA). Statistical significance was set to $\mathrm{p}$ value $<0.05$. The results are presented as mean value \pm standard deviation.

\section{Results and discussion}

\subsection{The role of the physico-chemical transition}

The proposed hydrogel synthesis protocol takes advantages of the chemical structure of the raw polymeric materials to define the optimal configuration of a 3D stable and biocompatible scaffold. The intermediate physical hydrogel represents a key point for the adequate spatial orientation, mobility and distribution of the polyacrylic acid and elastamine chains. In addition, it provides the condition to form stable covalent cross-linking in solid phase, without the use of any solvent. Carbomer is a branched polyacrylic acid characterized by side-chain carboxyl groups that cause the formation of a gel-like solution, according to the increased viscosity, when the polymer is dissolved in water. The resulting high viscous consistency is correlated to the carbomer ability to absorb and retain water; the polymer chains are involved in the interactions with the water self-ionization that provides hydroxide and hydronium ions: $-\mathrm{COOH}$ are deprotonated and the formation of carboxylate anions occurs. Carbomer also shows anti-inflammatory properties and high biocompatibility in therapeutic application and tissue engineering $[15,39,40]$. Used elastamine is a water soluble polyetherdiamine formed by propylene oxide and ethylene oxide, with terminal-chain primary amine groups; it is able to increase the hydrophilicity and the flexibility of the final material. In water, elastamine can be protonated, resulting in $-\mathrm{NH}_{3}{ }^{+}$terminal groups. When the polymer aqueous solutions are mixed with each other, the presence of anions and cations promotes ionic interactions, with a further viscosity increase and the formation of a physical gel system. The electrostatic bonds are the cause of the 3D carbomer-elastamine entanglement but, due to their nature, they can be easily disrupted when the hydrogel is submerged in water or PBS solution, because the added ions interfere with the physical gel cross-linking and the structure simply dissolves.

For these reasons, the freeze-drying approach allows preserving the hydrogel structure and the subsequent microwave irradiation ensures the formation of covalent bonds, only exploiting the carboxyl and amine moiety, that represent strong interconnections of the polymeric meshes. The transition from physical to chemical regime occurs in solid-state without the introduction of any solvent, polymer chain functionalization or specific/critical experimental conditions and is the keystone for the design of biocompatible antimicrobial scaffolds without introducing tailored antibacterial molecules (the use of which would require additional synthesis steps, cost or care). A schematic representation of the discussed strategy and the putative structures of the physical and chemical (with amidic bonds) hydrogels are illustrated in Fig. 1. 

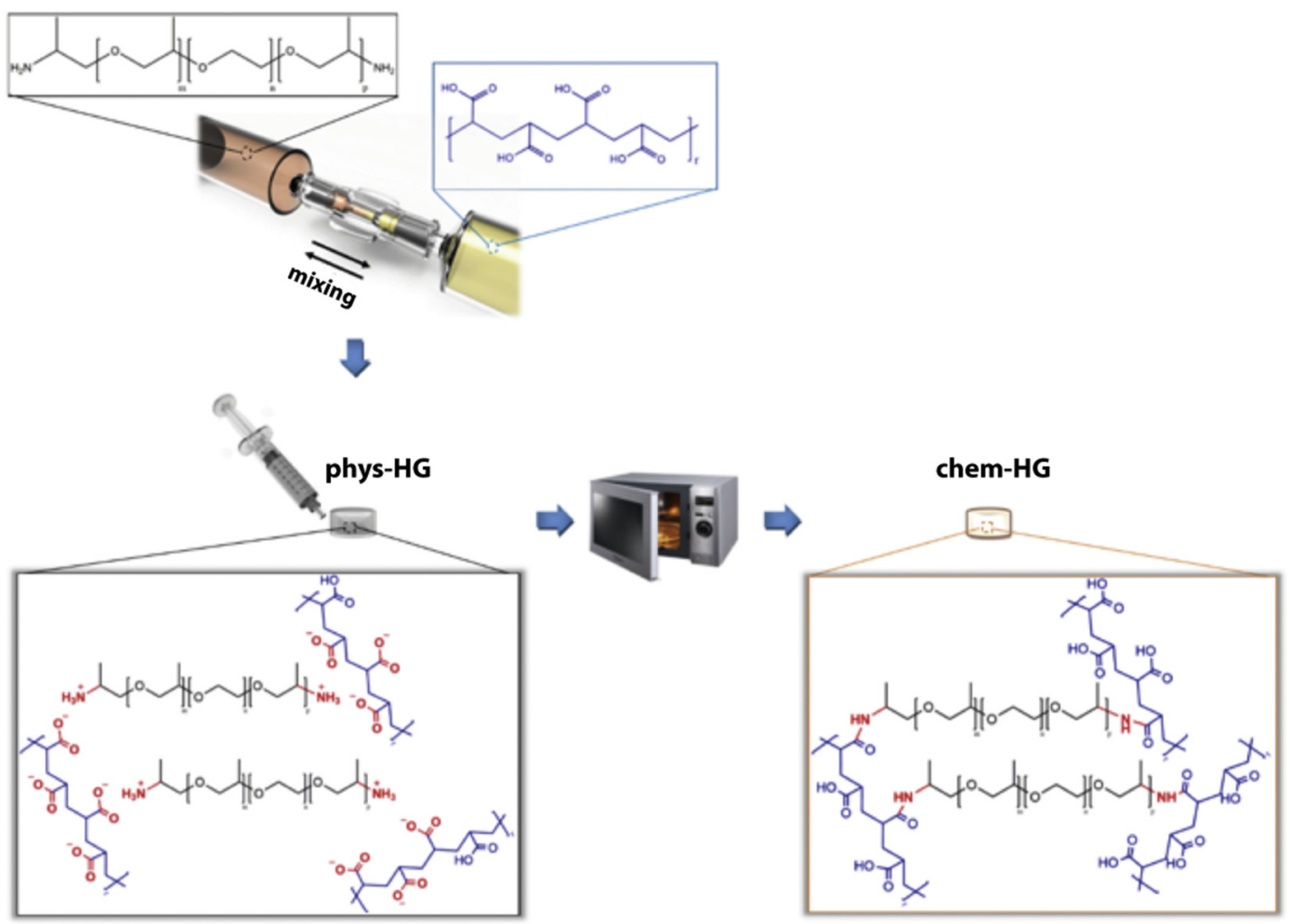

Fig. 1. Scheme of hydrogel synthesis exploiting the physico-chemical transition. In red are highlighted the electrostatic interactions between the ionic groups of carbomer and elastamine to give rise to phys-HG and the amidic bonds to form the crosslinked scaffold of chem-HG. (For interpretation of the references to color in this figure legend, the reader is referred to the web version of this article.)

The use of polyetherdiamines is detected in biomedical applications due to its in vitro and in vivo biocompatibility. For example, Javan and coworkers [41] introduced a jeffamine chemical functionalization in hyaluronic acid-based hydrogels to formulate a prolonged and sustained drug release and reduce the number of hydrogel injections: in vivo studies showed the efficiency of this material thanks to the improved biocompatibility and no signal of cell viability reduction. Similar biocompatibility aims were achieved in other works: the jeffamine was used as monomer to preserve some physico-mechanical properties, blood compatibility and cargo release [42-45], as copolymer with acrylamides derivatives to design 3D scaffolds able to modulate the lower critical solution temperature (LCST) and internalize cells [46] or as cross-linker to satisfy the no-cytotoxicity criteria [47]. However, the evaluation of the polymer antimicrobial features and the design of a 3D scaffold able to preserve them is not widely investigated.

\subsection{Material characterization}

Amidic bonds are demonstrative of the intermolecular association between carbomer and elastamine chains and the formation of a chemical network. Especially in the amidation reactions, the use of microwave irradiation is defined as a comfortable technique compared to the common way of synthesis $[48,49]$. The main benefits of microwaveassisted polymer synthesis are the reduced reaction time, the absence of organic solvents, the limitation of side reactions and the removal of unreacted species. In this work, the direct irradiation of polyacrylic acid and amine groups was efficient enough to obtain the desired product in a high yield after a short reaction time. The chemical characterization of the hydrogel was performed through FT-IR analysis. Moreover, the FT-IR spectroscopy could also provide information about the amide formation, noting the evolution of the corresponding absorption band. In this way, it was possible to draw the conversion-time chart at different time points.

Fig. 2 shows the FT-IR spectra of phys-HG $(A)$ and chem-HG at fixed time points ( $B=5 \mathrm{~min}, C=15 \mathrm{~min}, D=25 \mathrm{~min}$ ). In all spectra the polymers characteristic bands are detectable: with regards to carbomer, the wavenumbers range $3650-3300 \mathrm{~cm}^{-1}$ shows the stretching vibration of $-\mathrm{OH}$ residual groups, at $2950 \mathrm{~cm}^{-1}$ the $\mathrm{C}-\mathrm{H}$ chain stretching is recorded, whereas the $\mathrm{C}=\mathrm{O}$ stretching band is detectable around $1655 \mathrm{~cm}^{-1}$. The $-\mathrm{CH}_{2}$ narrow peak is visible at $1435-1300 \mathrm{~cm}^{-1}$ and the signal at $1256 \mathrm{~cm}^{-1}$ is associated to the $\mathrm{C}-\mathrm{O}$ vibrations [50,51]. The elastamine polyether backbone $(\mathrm{C}-\mathrm{O}-\mathrm{C})$ is well visible around $1100 \mathrm{~cm}^{-1}$ (symmetric and asymmetric stretching [52]) and the $\mathrm{C}-\mathrm{H}$ stretch is around $2870 \mathrm{~cm}^{-1}$, whereas the amine end groups are recognizable as weak signal at $3225 \mathrm{~cm}^{-1}$. The other signals in the wavenumber range $1500-500 \mathrm{~cm}^{-1}$ are generally related to combination of vibrations, bending, scissoring and deformation of $-\mathrm{CH}_{2}$ and $-\mathrm{CH}_{3}$ moieties [53].

The amide bond (*) is clearly detectable at $1713 \mathrm{~cm}^{-1}$ : in particular, chem-HG FT-IR spectra show the signal increase at different time points, confirming the reaction between carboxyl and amine groups. According to this trend, amide formation could be evaluated over time as percentage of covalent bond within the hydrogel scaffold [54]. The appearance of the peak at $1713 \mathrm{~cm}^{-1}$, which does not exist in the samples before microwave irradiation, was used to evaluate the conversion. The peak at $1100 \mathrm{~cm}^{-1}$ was chosen as an internal standard because representative of an invariant and well-defined group (elastamine polyether chains) and all spectra were signal-normalized to this reference. Absorbances were correlated to the IR peak intensity and the conversion of the reactive groups to amide was determined by the Lambert-Beer law $[55,56]$ from the normalized changes of absorbance at $1713 \mathrm{~cm}^{-1}\left(\mathrm{~A}_{\mathrm{am}}\right)$ scaled to the elastamine area at $1100 \mathrm{~cm}^{-1}\left(\mathrm{~A}_{\mathrm{ref}}\right)$, as reported in Eq. (2) (Supporting Information):

$\alpha_{\%}=\frac{\mathrm{A}_{\mathrm{am}}}{\mathrm{A}_{\mathrm{ref}}} \cdot 100$

where with $\alpha_{\%}$ the amount of formed bond was indicated. It was experimentally evaluated that not all the elastamine was involved in the 
D

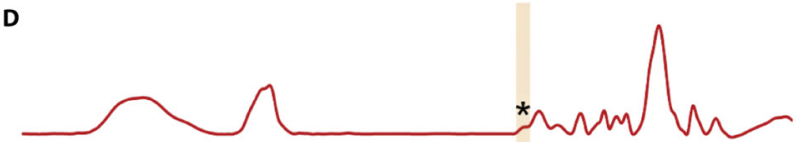

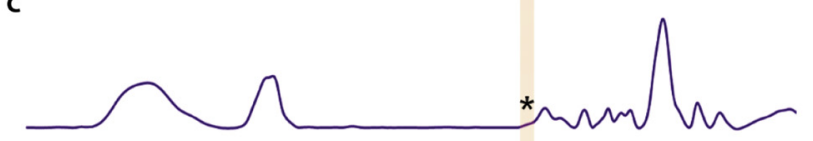

B



A



E

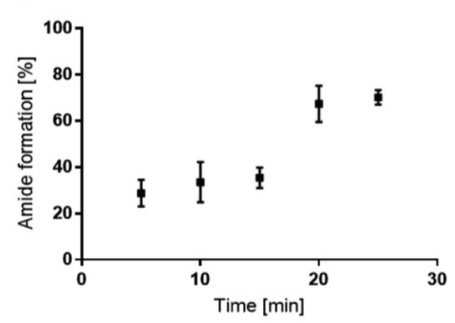

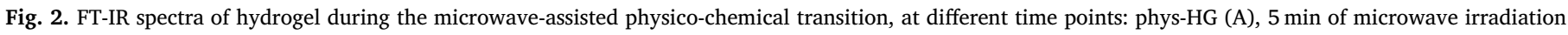
(B), 15 min of microwave irradiation (C) and $25 \mathrm{~min}$ of microwave irradiation (D) corresponding to final chem-HG; (E) plot of amide bond formation over time.

reaction, but only the $72.5 \%$ (Supporting Information): as a consequence, the obtained amide bond percentage was calculated and reported in the plot according to this consideration. The performed investigation showed that our hydrogels were characterized by covalent bonds, but the presence of residual elastamine amine groups (that is the limiting reagent) indicated continuing electrostatic interactions between $-\mathrm{COOH}$ and $-\mathrm{NH}_{2}$. For this reason, we classified the final network as physico-chemical hydrogel and we previously discussed the physical to chemical transition step. The amide formation trend (Fig. 2, pointed out in yellow) highlights a progressive microwave-assisted reaction, with a slow increase in the range $5-20 \mathrm{~min}$ (from $28 \%$ to $35.5 \%$ ) and the achievement of the final polymer interconnections at $25 \mathrm{~min}$ (72.5\%).

This behavior could be explained considering the hydrogel structure and the microwave mean free path: at first, the reaction occurred at the phys-HG surface, then the irradiation was able to achieve the functional groups inside the meshes and gave rise to covalent linkages in the inner core. In addition, the samples were subjected to the electromagnetic stimulation for further periods of time but, as showed, already at $30 \mathrm{~min}$ the reaction did not move forward (71.2\%), indicating the achievement of an amide formation plateau; with longer times, the hydrogel underwent thermos-oxidative degradation and collapsed.

\subsection{Hydrogel physical properties}

Rheological studies were carried out to characterize the elastic/ viscous hydrogel behavior. In particular, a comparison between the rheology of phys-HG and chem-HG was conducted in order to investigate the potential effect of the microwave irradiation and the physico-chemical transition in the network. The obtained data are showed in Fig. 3.

The storage modulus $\mathrm{G}^{\prime}$ was found to be approximately one order of magnitude higher than the corresponding loss modulus $\mathrm{G}^{\prime \prime}$ both in physHG and in chem-HG samples, with a frequency-dependent behavior at low frequencies: physical gels were characterized by $\mathrm{G}^{\prime}=1490 \mathrm{~Pa}$ and $\mathrm{G}^{\prime \prime}=372 \mathrm{~Pa}$ at $10 \mathrm{rad} / \mathrm{s}$ and by $\mathrm{G}^{\prime}=780 \mathrm{~Pa}$ and $\mathrm{G}^{\prime \prime}=145 \mathrm{~Pa}$ at $0.1 \mathrm{rad} / \mathrm{s}$, whereas the amide-based samples recorded a variation of $\mathrm{G}^{\prime}$ from $5160 \mathrm{~Pa}$ to $4380 \mathrm{~Pa}$ and $\mathrm{G}^{\prime \prime}$ from $545 \mathrm{~Pa}$ to $680 \mathrm{~Pa}$, in the same frequency sweep. The marked phys-HG storage modulus trend is detectable in colloidal gels and it is indicative of the physical nature and interconnections [57,58]; instead, $G^{\prime}$ in chem-HG exhibits frequency dependence only at low frequency values, below $0.5 \mathrm{rad} / \mathrm{s}$, which may be indicative of a reduction of the physical macromolecular interchain interactions and of the viscoelastic response of the system, matching the typical characteristic signature of a solid-like gel. The different order of magnitude observed for $\mathrm{G}^{\prime}$ and $\mathrm{G}^{\prime \prime}$ is also indicative of an elastic rather than viscous material. Moreover, the corresponding $\mathrm{G}^{\prime}$ and $\mathrm{G}^{\prime \prime}$ values in chem-HG are higher than in phys-HG, pointing out the elastic property and the improved stiffness due to the amide cross-linking. Through DSS test, the crossover strain can be evaluated as the value at which the contribution of the material damping $\tan (\delta)$ is predominant with respect to $G^{\prime}$. At low values of strain, the $\mathrm{G}^{\prime}$ trends of phys-HG (Fig. 3B) and chem-HG (Fig. 3C) indicate a network of packed polymeric chains and the correlation with $\tan (\delta)$ confirmed for both hydrogels liquid-like behaviors rather than a solid-like nature. Moreover, the intersection of $\mathrm{G}^{\prime}$ and $\mathrm{G}^{\prime \prime}$ occurs at lower values in chem-HG than in phys-HG (at shear stress $\tau=131 \mathrm{~Pa}$ and $\tau=345 \mathrm{~Pa}$ ) indicating more stiffness in the chemical network according to the presence of both amide and electrostatic cross-linking that probably create preferential break-lines compared to the physical gel under the same strain [59]. One hydrogel distinctive feature is the ability to retain high amount of water.

The hydrogel swelling occurs due to the polymer chain stretching during the exposure to aqueous solvents, counterbalanced by an elastic force acting in the opposite direction generated by increasing the elongation of the system. Chem-HG swelling behavior was investigated in distilled water and in PBS solution and the results are reported in Fig. 4A.

The same study was not feasible for phys-HG because in aqueous environment the physical bonds between carboxyl and amine groups became weaker due to the auto-dissociation of water or the addition of salts (PBS) that interfered and dissolved the gel in $<30 \mathrm{~min}$ (data not shown). The experimental results suggest that chem-HG exhibited fast swelling kinetics and their swelling equilibrium was reached within $4 \mathrm{~h}$. Moreover different swelling ratios were recorded in water (about 900\%) and in PBS solution (375\%).

The changed behavior in PBS could be considered related to the increased ionic strength and so ionic interactions between the salts and the polymeric chains: PBS dissociation is able to generate interactions with the oxygen and carboxyl moieties creating physical bindings that move closer the polymeric chains and result in a mesh reduction. This effect was not observed in samples immersed in distilled water: the amount of entrapped water was higher and limited only by the chains elongation and the cross-linking points. In summary, in both cases hydrogel swelling behavior was expressed, preserving the peculiar feature of the polymeric network and the presence of saline solution could affect the behavior due to the physico-chemical nature of the 


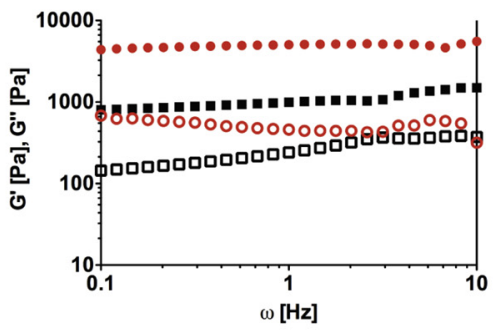



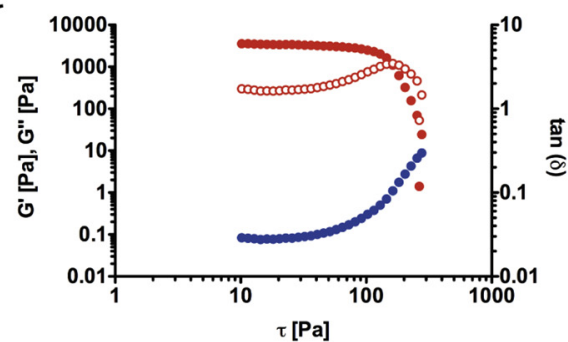



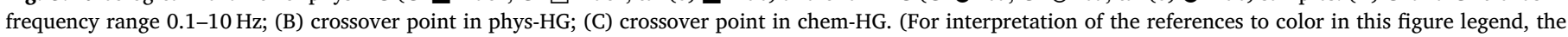
reader is referred to the web version of this article.)

system. Considering the potential application in conditions dissimilar from the neutral ones, the swelling studies were also performed in acid and alkaline environments; $\mathrm{pH}=3$ is representative of gastric conditions, whereas alkaline $\mathrm{pH}$ is indicative of pancreatic environment and fluid $(\mathrm{pH}=8.8)[60,61]$. Moreover, the study of hydrogels in low and high $\mathrm{pH}$ represents an approach to produce materials potentially useful for chronic wounds [62,63], for the re-alkalization of carbonated materials [64], for antitumoral administrations or to counteract undesired $\mathrm{pH}$ changes [60]. In this work, the swelling evaluation was conducted at pH 3 and 10 (Supporting Information) and compared the hydrogels state once they reached the equilibrium to the physiologic $\mathrm{pH} 7.4$. Fig. 4B illustrates the hydrogel swelling after $48 \mathrm{~h}$ : at acid $\mathrm{pH}$ the recorded value was $445 \%$, whereas in alkaline system was around $600 \%$. Both conditions exhibited lower swelling than in samples treated with distilled water and this could be explained considering the ions at stake. The polyacrylic acid $\mathrm{pK}_{\mathrm{a}}$ is reported to be 4.7 [65]. When the $\mathrm{pH}$ was less than $\mathrm{pK}_{\mathrm{a}}$ of PAA, the $\mathrm{H}^{+}$ionic strength was high and able to suppress the ionization of the carboxylic acid groups; as result, carbomer neutral chains were rather flexible due to their compact conformation [66].

The principal acid $\mathrm{pH}$ effect is related to the formation of hydrogen bindings (van der Waals forces) in the elastamine polyethoxylate moiety that reduces the mean free space among the elastamine and carbomer chains; as a result, a reduction of hydrogel meshes occurs and the swelling appeared reduced than in water. At $\mathrm{pH}=10$, the same effect of net mesh sizes constraint is due to the $\mathrm{Na}^{+}$(alkaline conditions are performed using $\mathrm{NaOH} 1 \mathrm{M}$ ) interactions with carbomer carboxylate ions. In all experimental procedures, hydrogel scaffolds were able to preserve their structural integrity over the investigated time. The morphology of the chem-HG was also investigated through SEM analysis, as reported in Fig. 5.

The results revealed that dried samples possess a highly entangled structure, with small and big pores in the diameter range $70-270 \mu \mathrm{m}$, that seem to define a sponge-like state. In addition, most of the pores

A



are interconnected. This demonstrates their microscopic porous structure with a complex 3D construction.

\subsection{Antimicrobial results}

For this study, we used $S$. aureus and $C$. albicans as representative of biofilm-producing organisms and opportunistic pathogens. Both microorganisms were grown adsorbed on hydrogels. The in vitro assay revealed the chem-HG to be the most effective in microbial inhibition. Indeed, after $24 \mathrm{~h}$ incubation (Fig. 6), we observed a dramatic inhibition of $S$. aureus growth ( $>99 \%$ reduction in CFUs count, $\mathrm{p}=0.0159$ ) and a significant reduction (about $30 \%, \mathrm{p}=0.015$ ) for C. albicans, compared with controls (growth in absence of hydrogels).

\subsection{Drug release}

Once demonstrated the antimicrobial property of the synthetized hydrogels, their application as drug carriers was studied. The aim is the approval of the fundamental controlled drug delivery principles and offering the potential combination of therapeutic curative and sterile effects in only one scaffold.

The developed method is based on the evaluation of mimetic drugs release considering the steric hindrance or using a cleavable ester linker that temporarily graft the molecule to the hydrogel. Release studies were performed at $37{ }^{\circ} \mathrm{C}$, at physiologic $\mathrm{pH}$ that mimic the classic in vitro and in vivo environment. As previously assessed, SF was chosen to represent the behavior of drugs in salt form, able to dissociate in anions and cations in aqueous system, like corticosteroids and anti-inflammatory drugs [31,67], whereas $\mathrm{RhB}$ resembles the characteristics of carbonyl-based drugs as those involved in the treatment of cancer or spinal cord injury $[15,68,69]$.

\subsubsection{Release by steric hindrance}

$\mathrm{SF}$ and $\mathrm{RhB}$ were loaded within the hydrogels during the physical

B

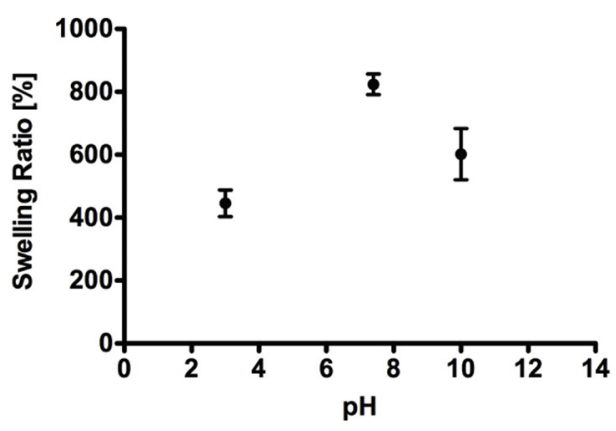



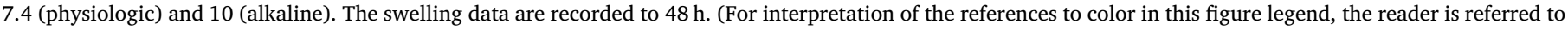
the web version of this article.) 

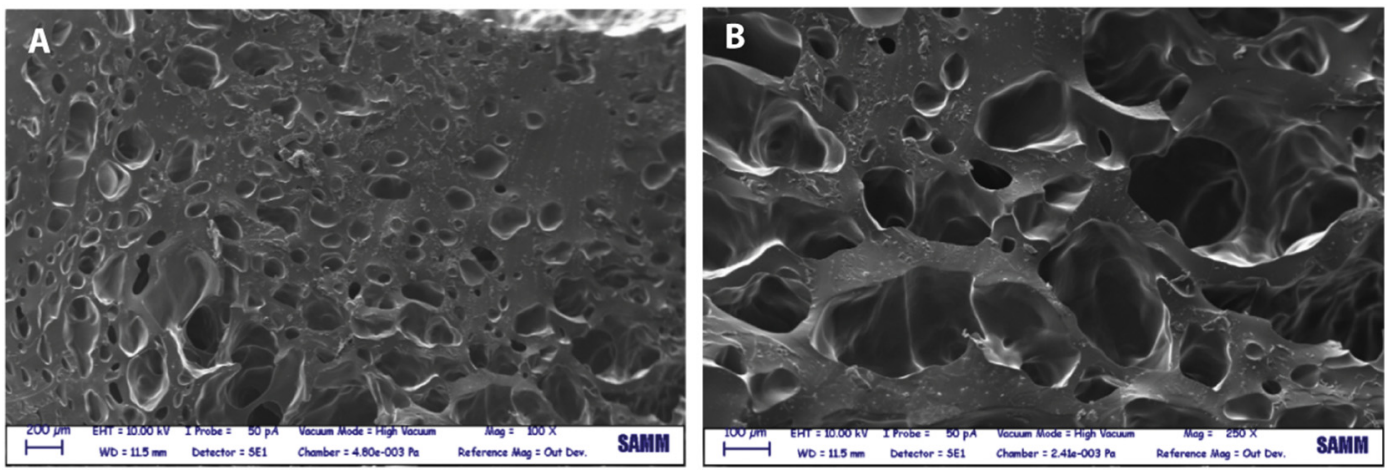

Fig. 5. SEM imagines of chem-HG hydrogels after freeze-drying at amplification $100 \times(A)$ and $250 \times(B)$.

formation of the 3D network. The different drug concentrations are evaluated according to the general concentration range proposed in hydrogels field and the solubility limit in water. However, the percentage drug release depends on drug concentration gradient generating the diffusion flow and it was normalized respect to the mass loaded (percentage), so that the kinetics became concentration independent [70]. The percentage of SF and RhB released was defined as the ratio between the released amount in the aqueous media and the total amount entrapped by steric hindrance within the hydrogel. Fig. 7 shows the $\mathrm{SF}$ and $\mathrm{RhB}$ release profiles at $\mathrm{pH}$ values 7.4 (A, D), 3 (B, E) and $10(\mathrm{C}, \mathrm{F})$.

Considering the neutral release environment, $\mathrm{SF}$ release is faster than $\mathrm{RhB}$ in the first hours (after $48 \mathrm{~h}$, the percentage of released SF is $80 \%$, whereas for $\mathrm{RhB} 60 \%$ ) and similar amounts of released drug are recorded only after $250 \mathrm{~h}$. The different trends are related to the electrostatic and ionic interactions with the polymer chains: the presence of fluorescein anions causes repulsion with the dominant carboxylate moieties and the mimetic drug comes out from the meshes more quickly than $\mathrm{RhB}$, which is neutral.

These repulsive effects overlap the attractive interaction between residual amine groups and the fluorescein anion. SF indeed is present in salt form at basic $\mathrm{pH}$ and its solubility is higher respect to acid and neutral pH. Salt form of SF resembles the behavior of many drugs saled in salt form like ibuprofene and ketoprofene. On the other hand, the $\mathrm{RhB}$ delivery rate is minimally influenced by the scaffold. In both cases, the hydrogels are able to sustain drug release, without introducing any polymeric modification. The influence of the system in delivering SF or $\mathrm{RhB}$ can be evaluated plotting release percentage against time square root, as showed in Fig. 7D. A linear plot is indicative of Fickian diffusion and the $y$-axis intercept value is indicative of the burst release. The condition of ideal controlled release system is characterized by linear trend during time and its y-axis intercept equal to zero [31,71]. Both $\mathrm{RhB}$ and SF release highlight linear trends in the first $8 \mathrm{~h}$, approaching then plateau trends. The SF burst release value is higher (40\%) than
RhB (19\%) according to the SF ionic interactions with the polymeric scaffold that facilitates the quick release of small hydrophilic molecules in the aqueous medium; on the other hand, the ability to control the drug release is slightly improved in the $\mathrm{RhB}$ case due to the absence anion-cation interactions. The drug releases in acid and basic conditions are characterized by different trends, compared to $\mathrm{pH}$ 7.4. In particular, at $\mathrm{pH} 3 \mathrm{RhB}$ release profile is faster and it is almost completed after $72 \mathrm{~h}$, while the escaped SF is $33 \%$ at the same time point suggesting a prolonged release over a longer period of time. In this condition, the high concentration of $\mathrm{H}^{+}$in the release medium counteracts the negative charge of carboxyl groups reducing the repulsion against fluorescein anions and promoting the attractive effect related to the elastamine protonation: as result, the SF release is delayed. The same electrostatic re-organization occurs in sample containing $\mathrm{RhB}$, but the mimetic drug is characterized by a slightly positive charge at $\mathrm{pH} 3$ and the repulsion with the polymeric scaffold encourages a modest enlargement of the meshes and a quick release of drug molecules. In alkaline aqueous medium, the release profiles are reversed: SF comes out faster than $\mathrm{RhB}$, due to the high repulsion between the drug anions and the carboxylate groups.

As discussed in the evaluation of hydrogel swelling behavior, the presence of $\mathrm{COO}^{-}$allows the elongation of the polymeric chains increasing the free spaces in the scaffold and the improved release of the loaded drug. This also explains the increased amount of released RhB compared to the corresponding study at $\mathrm{pH} 7.4$, considering that $\mathrm{RhB}$ is neutral at basic $\mathrm{pH}$ and no ionic interactions arise. The linear plots at $\mathrm{pH} 3$ and 10 show that the Fickian diffusion regime is well visible in the first $8 \mathrm{~h}$, then the drugs release reaches a plateau. In particular, at $\mathrm{pH} 3$, the SF burst release contribution is extremely small (1\%) suggesting the ability of the synthetized gels to control the release of salt form-drugs in acid conditions. This effect is less visible in the RhB samples, where the burst release value is about $20 \%$. In alkaline environment these release behaviors are reversed: RhB presents $15 \%$ of burst release (comparable to the neutral release environment), whereas SF about 36\%. The RhB
A



B

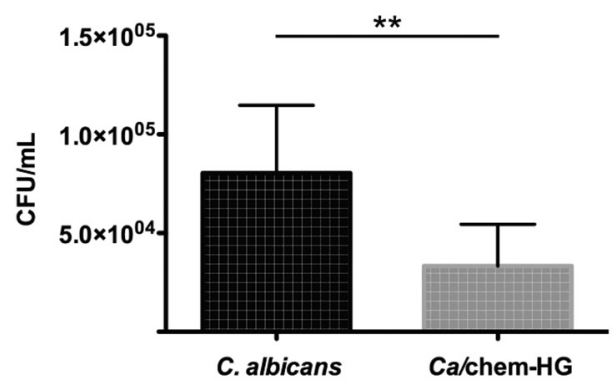

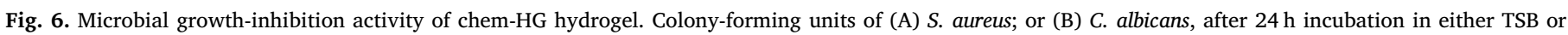

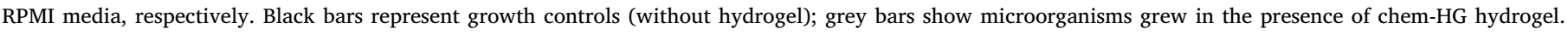
Values represent the mean of two independent experiments for each strain. Pairwise lines denote statistical significance; ${ }^{*} \mathrm{p}<0.05 ; * * \mathrm{p}<0.01$. 
A
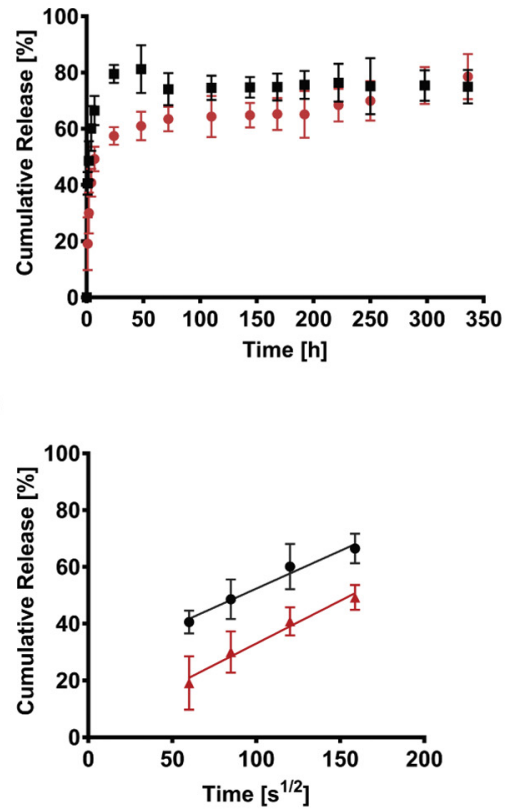

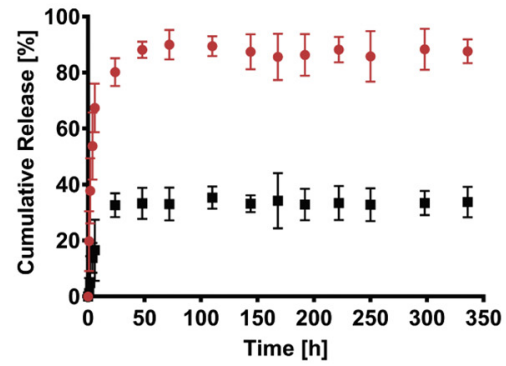

$\mathbf{E}$

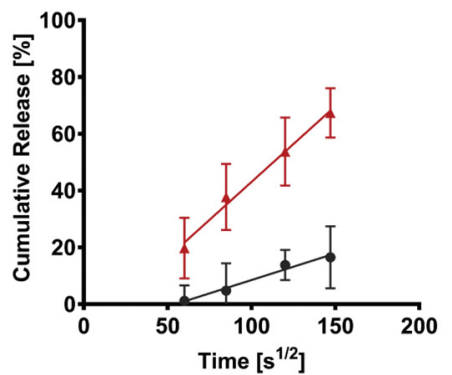

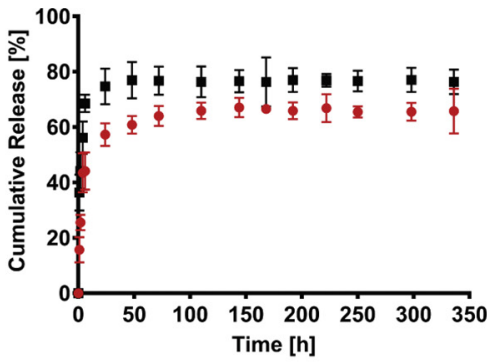

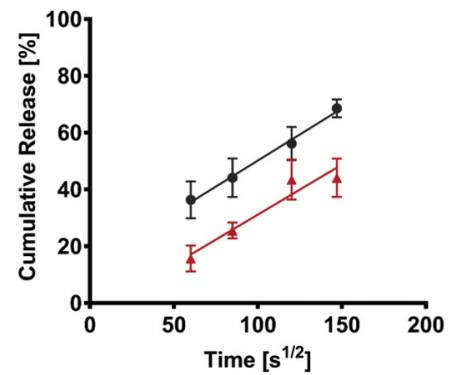

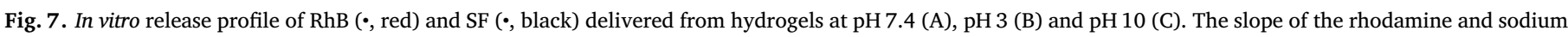

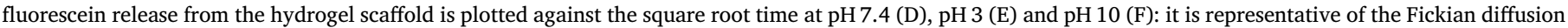



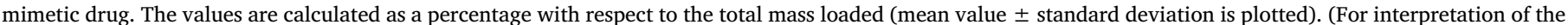
references to color in this figure legend, the reader is referred to the web version of this article.)

non-ionic nature allows a drug release not affected by the chemical behaviors of the carbomer and elastamine, which otherwise tune the SF escape from the network meshes.

\subsubsection{Release by ester hydrolysis}

These hydrogels were also tested as tunable drug delivery systems through the introduction of PEG carrying RhB through ester bond. The choice of a modified polymer instead of the direct chemical modification of the polymeric network is related to the intention to preserve the elastamine component, avoiding any reaction that could affect its properties. PEG reacted with carbomer carboxyl groups to bind to the scaffold in a stable manner [38] (IR, Supporting information) and the ester-RhB cleavability was investigated. The evaluations were performed, also in this case, at acid, neutral and alkaline $\mathrm{pH}$. Fig. 8 shows the amount of released RhB over time: at pH 7.4, only $30 \%$ of entrapped mimetic drug was escaped after $250 \mathrm{~h}$, indicating that the release kinetic is prolonged and dependent on the ester hydrolysis, which delay the release associated to this strategy.
The same trend is observed in the $\mathrm{RhB}$ release at $\mathrm{pH} 10$, whereas in acid medium, there is a slight increase in the released amount due to the ester acid hydrolysis.

The linear trends plotting release percentage against $s^{1 / 2}$ are indicative of increased performances in terms of pure diffusive mass transport and of burst release contribution that results to be very small. In particular, it is recognizable a double diffusion regime with different slopes. The transition of the two regimes depends on the allocation of ester-RhB bond: when it is near the hydrogel/medium interface it can be more easily hydrolyzed (regime $i$ ) than the linker present in the inner core (regime ii). The obtained results make possible to state that the proposed hydrogels can be used as drug carriers also introducing cleavable linkers designing a system characterized by a delayed drug release with consequent higher performance with respect to hydrogels where drugs are only physically entrapped.

B

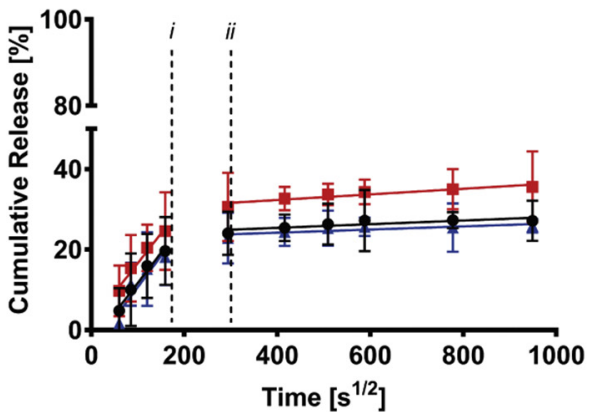

Fig. 8. A: In vitro release profile of RhB linked to the hydrogels scaffold through ester bond, at pH 7.4 ( $\bullet$, black), $\mathrm{pH} 3$ ( $\mathbf{\square}$, red) and pH 10 ( $\boldsymbol{\Delta}$, blue). B: Evaluation of $\mathrm{RhB}$ cumulative release percentage against $\mathrm{s}^{1 / 2}$ at the different $\mathrm{pH}$ : the linear trends are representative of the Fickian diffusion regime of the drug from the hydrogel ( $p<0.0001$ between all the groups), according to the ester cleavability. The values are calculated as a percentage with respect to the total mass loaded (mean value \pm standard deviation is plotted). (For interpretation of the references to color in this figure legend, the reader is referred to the web version of this article.) 


\section{Conclusions}

This work proposes the design of innovative hydrogel systems using a simple synthesis approach based on a physical-chemical transition that exploits the physico-chemical interactions among carbormer and elastamine, preserving their peculiar properties. The main advantage is related to the non-use of inorganic particles, antibacterial agents or antibiotics to perform a suitable antimicrobial effect, overcoming all questionable aspects related to the cytotoxicity or the concentration of the active molecules to avoid side-effects: here, the antimicrobial activity is due to the polymeric components. Moreover, the hydrogels are able to preserve their structure in environments at different $\mathrm{pH}$ and could be used as controlled drug delivery systems. In particular, thanks to the nature and the polymeric chains spatial organization, it is possible to prepare drug carriers with drug loading and release via steric hindrance or polymer functionalization, offering a wide range of release kinetics, that can be chosen according to the needs of the final therapeutic application. The opportunity to synthetize a system able to perform the double effect of antimicrobial and drug delivery is a pivotal challenge to reduce the number of administrations, injections or dosing in biomedical fields, and these scaffolds appear as a promising tool. Finally, the antimicrobial property could be useful in other fields, such as for environmental and industrial applications, and this shows the potential of this material.

\section{Appendix A. Supplementary data}

Supplementary data to this article can be found online at https:// doi.org/10.1016/j.msec.2019.109791.

\section{References}

[1] K.E. Boehle, J. Gilliand, C.R. Wheeldon, A. Holder, J.A. Adkins, B.J. Geiss, et al., Utilizing paper-based devices for antimicrobial-resistant bacteria detection, Angew Chem Int Edit 56 (2017) 6886-6890.

[2] B. Nabil, E. Ahmida, C. Christine, V. Julien, A. Abdelkrim, Polyfunctional cotton fabrics with catalytic activity and antibacterial capacity, Chem. Eng. J. 351 (2018) 328-339.

[3] F.J. Rodriguez, A. Torres, A. Penaloza, H. Sepulveda, M.J. Galotto, A. Guarda, et al., Development of an antimicrobial material based on a nanocomposite cellulose acetate film for active food packaging, Food Addit Contam A 31 (2014) 342-353.

[4] C.M. Gonzalez-Henriquez, M.A. Sarabia-Vallejos, J. Rodriguez-Hernandez, Advances in the fabrication of antimicrobial hydrogels for biomedical applications, Materials 10 (2017).

[5] W.W. Gao, Y.J. Chen, Y. Zhang, Q.Z. Zhang, L.F. Zhang, Nanoparticle-based local antimicrobial drug delivery, Adv Drug Deliver Rev 127 (2018) 46-57.

[6] J. Hoque, B. Bhattacharjee, R.G. Prakash, K. Paramanandham, J. Haldar, Dual function injectable hydrogel for controlled release of antibiotic and local antibacterial therapy, Biomacromolecules 19 (2018) 267-278.

[7] M.H. Xiong, Y. Bao, X.Z. Yang, Y.H. Zhu, J. Wang, Delivery of antibiotics with polymeric particles, Adv Drug Deliver Rev 78 (2014) 63-76.

[8] F.S. Taccone, O. Bond, F.Z. Cavicchi, M. Hites, Individualized antibiotic strategies, Curr Opin Anesthesio 29 (2016) 166-171.

[9] T.W. Felton, W.W. Hope, J.A. Roberts, How severe is antibiotic pharmacokinetic variability in critically ill patients and what can be done about it? Diagn Micr Infec Dis 79 (2014) 441-447.

[10] S. Sattari, A.D. Tehrani, M. Adeli, pH-responsive hybrid hydrogels as antibacterial and drug delivery systems, Polymers-Basel 10 (2018).

[11] W.G. Xu, S.J. Dong, Y.P. Han, S.Q. Li, Y. Liu, Hydrogels as antibacterial biomaterials, Curr Pharm Design 24 (2018) 843-854.

[12] S.Q. Li, S.J. Dong, W.G. Xu, S.C. Tu, L.S. Yan, C.W. Zhao, et al., Antibacterial hydrogels, Adv Sci 5 (2018).

[13] A.O. Adebisi, B.R. Conway, Modification of drug delivery to improve antibiotic targeting to the stomach, Ther. Deliv. 6 (2015) 741-762.

[14] Q.F. Wang, H.J. Yu, L. Zhong, J.Q. Liu, J.Q. Sun, J.C. Shen, Incorporation of silver ions into ultrathin titanium phosphate films: in situ reduction to prepare silver nanoparticles and their antibacterial activity, Chem. Mater. 18 (2006) 1988-1994.

[15] G. Perale, F. Rossi, M. Santoro, M. Peviani, S. Papa, D. Llupi, et al., Multiple drug delivery hydrogel system for spinal cord injury repair strategies, J. Control. Release 159 (2012) 271-280.

[16] N. Annabi, A. Tamayol, J.A. Uquillas, M. Akbari, L.E. Bertassoni, C. Cha, et al., 25th anniversary article: rational design and applications of hydrogels in regenerative medicine, Adv. Mater. 26 (2014) 85-124.

[17] R.R. Choudhury, J.M. Gohil, S. Mohanty, S.K. Nayak, Antifouling, fouling release and antimicrobial materials for surface modification of reverse osmosis and nanofiltration membranes, J. Mater. Chem. A 6 (2018) 313-333.
[18] P.M. Nguyen, N.S. Zacharia, E. Verploegen, P.T. Hammond, Extended release antibacterial layer-by-layer films incorporating linear-dendritic block copolymer micelles, Chem. Mater. 19 (2007) 5524-5530.

[19] A.S. Veiga, J.P. Schneider, Antimicrobial hydrogels for the treatment of infection, Biopolymers 100 (2013) 637-644.

[20] D. Sun, M.B. Shahzad, M. Li, G. Wang, D. Xu, Antimicrobial materials with medical applications, Mater. Technol. 30 (2015) (B90-B5).

[21] X. Yi, J.P. He, X.L. Wang, Y. Zhangg, G.X. Tan, Z.N. Zhou, et al., Tunable mechanical, antibacterial, and cytocompatible hydrogels based on a functionalized dual network of metal coordination bonds and covalent crosslinking, Acs Appl Mater Inter 10 (2018) 6190-6198.

[22] H. Tang, A. Lu, L. Li, W.J. Zhou, Z.X. Xie, L.N. Zhang, Highly antibacterial materials constructed from silver molybdate nanoparticles immobilized in chitin matrix, Chem. Eng. J. 234 (2013) 124-131.

[23] I. Irwansyah, Y.Q. Li, W.X. Shi, D.P. Qi, W.R. Leow, M.B.Y. Tang, et al., Grampositive antimicrobial activity of amino acid-based hydrogels, Adv. Mater. 27 (2015) 648-654.

[24] A.L. Lakes, R. Peyyala, J.L. Ebersole, D.A. Puleo, J.Z. Hilt, T.D. Dziubla, Synthesis and characterization of an antibacterial hydrogel containing covalently bound vancomycin, Biomacromolecules 15 (2014) 3009-3018.

[25] Y. Lee, K.H. Choi, K.M. Park, J.M. Lee, B.J. Park, K.D. Park, In situ forming and $\mathrm{H}_{2} \mathrm{O}_{2}$-releasing hydrogels for treatment of drug-resistant bacterial infections, Acs Appl Mater Inter 9 (2017) 16891-16900.

[26] D. Chudobova, S. Dostalova, B. Ruttkay-Nedecky, R. Guran, M.A.M. Rodrigo, K. Tmejova, et al., The effect of metal ions on Staphylococcus aureus revealed by biochemical and mass spectrometric analyses, Microbiol. Res. 170 (2015) 147-156.

[27] Y.M. Wan, L.B. Liu, S.S. Yuan, J. Sun, Z.B. Li, pH-responsive peptide supramolecular hydrogels with antibacterial activity, Langmuir 33 (2017) 3234-3240.

[28] I. Caron, F. Rossi, S. Papa, R. Aloe, M. Sculco, E. Mauri, et al., A new three dimensional biomimetic hydrogel to deliver factors secreted by human mesenchymal stem cells in spinal cord injury, Biomaterials 75 (2016) 135-147.

[29] M. Barbosa, M. Martins, P. Gomes, Grafting techniques towards production of peptide-tethered hydrogels, a novel class of materials with biomedical interest, Gels 1 (2015) 194.

[30] J. Sestak, M. Mullins, L. Northrup, S. Thati, M.L. Forrest, T.J. Siahaan, et al., Singlestep grafting of aminooxy-peptides to hyaluronan: a simple approach to multifunctional therapeutics for experimental autoimmune encephalomyelitis, $\mathrm{J}$. Control. Release 168 (2013) 334-340.

[31] E. Mauri, G.M.F. Chincarini, R. Rigamonti, L. Magagnin, A. Sacchetti, F. Rossi, Modulation of electrostatic interactions to improve controlled drug delivery from nanogels, Mat Sci Eng C-Mater 72 (2017) 308-315.

[32] T. Casalini, M. Salvalaglio, G. Perale, M. Masi, C. Cavallotti, Diffusion and aggregation of sodium fluorescein in aqueous solutions, J. Phys. Chem. B 115 (2011) $12896-12904$.

[33] H. Sun, J. Chen, X. Han, H.L. Liu, Multi-responsive hydrogels with UCST- and LCSTinduced shrinking and controlled release behaviors of rhodamine B, Mat Sci Eng CMater 82 (2018) 284-290.

[34] B.S. Kim, Y.C. Shin, pH-sensitive swelling and release behaviors of anionic hydrogels for intelligent drug delivery system, J. Appl. Polym. Sci. 105 (2007) 3656-3661.

[35] R.W. Ramette, E.B. Sandell, Rhodamine B Equilibria, J. Am. Chem. Soc. 78 (1956) $4872-4878$.

[36] R.Y. Zhang, M. Hummelgard, G. Lv, H. Olin, Real time monitoring of the drug release of rhodamine B on graphene oxide, Carbon 49 (2011) 1126-1132.

[37] M. Obata, M. Morita, K. Nakase, K. Mitsuo, K. Asai, S. Hirohara, et al., Synthesis and photophysical properties of rhodamine B dye-bearing poly(isobutyl methacrylateco-2,2, 2-trifluoroethyl methacrylate) as a temperature-sensing polymer film, J Polym Sci Pol Chem 45 (2007) 2876-2885.

[38] E. Mauri, F. Rossi, A. Sacchetti, Tunable drug delivery using chemoselective functionalization of hydrogels, Mat Sci Eng C-Mater 61 (2016) 851-857.

[39] N.A. Peppas, J.B. Thomas, J. McGinty, Molecular aspects of mucoadhesive carrier development for drug delivery and improved absorption, J Biomat Sci-Polym E 20 (2009) 1-20.

[40] Y. Zheng, W.Q. Ouyang, Y.P. Wei, S.F. Syed, C.S. Hao, B.Z. Wang, et al., Effects of Carbopol (R) 934 proportion on nanoemulsion gel for topical and transdermal drug delivery: a skin permeation study, Int. J. Nanomedicine 11 (2016) 5971-5987.

[41] N.B. Javan, H. Montazeri, L.R. Shirmard, N.J. Omid, G.R. Barbari, M. Amini, et al., Preparation, characterization and in vivo evaluation of a combination delivery system based on hyaluronic acid/jeffamine hydrogel loaded with PHBV/PLGA blend nanoparticles for prolonged delivery of Teriparatide, Eur. J. Pharm. Sci. 101 (2017) 167-181.

[42] T.Y. Lo, Y.J. Wang, D.M. Liu, W.T. Whang, Surface characteristics and biofunctionality of a novel high-performance, hydrophilic Jeffamine-added fluorocontaining polyimide for biomedical applications, J. Polym. Res. 22 (2015).

[43] A.S. Erturk, M.U. Gurbuz, M. Tulu, New-generation Jeffamine (R) D230 core amine, TRIS and carboxyl-terminated PAMAM dendrimers: synthesis, characterization and the solubility application for a model NSAID drug Ibuprofen, Marmara Pharm J 21 (2017) 385-399.

[44] K. Ozturk, A.S. Erturk, C. Sansozen, M. Tulu, S. Calis, Cytotoxicity and in vitro characterization studies of synthesized Jeffamine-cored PAMAM dendrimers, J. Microencapsul. 31 (2014) 127-136.

[45] A. Zeynep, A. Fahri, S. Mehmet, K.S. Naci, Evaluation of Jeffamine ${ }^{\oplus}$-cored PAMAM dendrimers as an efficient in vitro gene delivery system, J. Biomed. Mater. Res. A 100A (2012) 2623-2628.

[46] J.M. Heffernan, J.B. McNamara, S. Borwege, B.L. Vernon, N. Sanai, S. Mehta, et al. PNIPAAm-co-Jeffamine (R) (PNJ) scaffolds as in vitro models for niche enrichment 
of glioblastoma stem-like cells, Biomaterials 143 (2017) 149-158.

[47] A. Erdem, F.A. Ngwabebhoh, U. Yildiz, Fabrication and characterization of soft macroporous Jeffamine cryogels as potential materials for tissue applications, RSC Adv. 6 (2016) 111872-111881.

[48] O. Kretschmann, S. Schmitz, H. Ritter, Microwave-assisted synthesis of associative hydrogels, Macromol Rapid Comm 28 (2007) 1265-1269.

[49] M. Iannelli, H. Ritter, Microwave-assisted direct synthesis and polymerization of chiral acrylamide, Macromol. Chem. Phys. 206 (2005) 349-353.

[50] J. Dong, Y. Ozaki, K. Nakashima, Infrared, Raman, and near-infrared spectroscopic evidence for the coexistence of various hydrogen-bond forms in poly(acrylic acid), Macromolecules 30 (1997) 1111-1117.

[51] M. Todica, R. Stefan, C.V. Pop, L. Olar, IR and Raman investigation of some poly (acrylic) acid gels in aqueous and neutralized state, Acta Phys. Pol. A 128 (2015) $128-135$.

[52] A. Sanchez-Ferrer, D. Rogez, P. Martinoty, Synthesis and characterization of new polyurea elastomers by sol/gel chemistry, Macromol. Chem. Phys. 211 (2010) $1712-1721$.

[53] V.D. Bermudez, L.D. Carlos, L. Alcacer, Sol-gel derived urea cross-linked organically modified silicates. 1. Room temperature mid-infrared spectra, Chem. Mater. 11 (1999) 569-580.

[54] X. Ramis, J.M. Salla, C. Mas, A. Mantecon, A. Serra, Kinetic study by FTIR, TMA, and DSC of the curing of a mixture of DGEBA resin and gamma-butyrolactone catalyzed by ytterbium triflate, J. Appl. Polym. Sci. 92 (2004) 381-393.

[55] C. Mas, X. Ramis, J.M. Salla, A. Mantecón, A. Serra, Copolymerization of diglycidyl ether of bisphenol A with $\gamma$-butyrolactone catalyzed by ytterbium triflate: shrinkage during curing, J. Polym. Sci. A Polym. Chem. 41 (2003) 2794-2808.

[56] J.M. Parnis, K.B. Oldham, Beyond the Beer-Lambert law: the dependence of absorbance on time in photochemistry, J. Photochem. Photobiol. A Chem. 267 (2013) 6-10.

[57] M. Wang, H.H. Winter, G.K. Auernhammer, Time and frequency dependent rheology of reactive silica gels, J Colloid Interf Sci 413 (2014) 159-166.

[58] C.Q. Yan, D.J. Pochan, Rheological properties of peptide-based hydrogels for biomedical and other applications, Chem. Soc. Rev. 39 (2010) 3528-3540.

[59] V.V. Erramreddy, S. Tu, S. Ghosh, Rheological reversibility and long-term stability of repulsive and attractive nanoemulsion gels, RSC Adv. 7 (2017) 47818-47832.

[60] G.K. Schwalfenberg, The alkaline diet: is there evidence that an alkaline pH diet benefits health? J. Environ. Public Health 2012 (2012) 727630.

[61] L.R.N. Angeloco, G.C.A. de Souza, E.A. Romao, P.G. Chiarello, Alkaline diet and metabolic acidosis: practical approaches to the nutritional management of chronic kidney disease, J Renal Nutr 28 (2018) 215-220.

[62] J. Koehler, L. Verheyen, S. Hedtrich, F.P. Brandl, A.M. Goepferich, Alkaline poly (ethylene glycol)-based hydrogels for a potential use as bioactive wound dressings, J. Biomed. Mater. Res. A 105 (2017) 3360-3368.

[63] C.C. Deng, W.L.A. Brooks, K.A. Abboud, B.S. Sumerlin, Boronic acid-based hydrogels undergo self-healing at neutral and acidic pH, ACS Macro Lett. 4 (2015) $220-224$.

[64] A. Jung, O. Weichold, Preparation and characterisation of highly alkaline hydrogels for the re-alkalisation of carbonated cementitious materials, Soft Matter 14 (2018) 8105-8111.

[65] N.A. Peppas, S.L. Wright, Drug diffusion and binding in ionizable interpenetrating networks from poly(vinyl alcohol) and poly(acrylic acid), Eur. J. Pharm. Biopharm. 46 (1998) 15-29.

[66] N. Sheikh, L. Jalili, F. Anvari, A study on the swelling behavior of poly(acrylic acid) hydrogels obtained by electron beam crosslinking, Radiat. Phys. Chem. 79 (2010) 735-739.

[67] H.S. Jamwal, G.S. Chauhan, Designing silica-based hybrid polymers and their application in the loading and release of fluorescein as a model drug and diagnostic agent, Adv. Polym. Technol. 37 (2018) 411-418.

[68] Y.T. Kim, J.M. Caldwell, R.V. Bellamkonda, Nanoparticle-mediated local delivery of methylprednisolone after spinal cord injury, Biomaterials 30 (2009) 2582-2590.

[69] J.L. Arias, F. Linares-Molinero, V. Gallardo, A.V. Delgado, Study of carbonyl iron/ poly(butylcyanoacrylate) (core/shell) particles as anticancer drug delivery systems - loading and release properties, Eur. J. Pharm. Sci. 33 (2008) 252-261.

[70] Y. Fu, W.J. Kao, Drug release kinetics and transport mechanisms of non-degradable and degradable polymeric delivery systems, Expert Opin Drug Del 7 (2010) 429-444.

[71] K. Vulic, M.S. Shoichet, Tunable growth factor delivery from injectable hydrogels for tissue engineering, J. Am. Chem. Soc. 134 (2012) 882-885. 